\title{
Persuasion Knowledge Toolkit: Requirements Gathering with Designer
}

\author{
Aeni Zuhana Saidin \\ School of Computing \\ University of Dundee \\ A.Z.Saidin@dundee.ac.uk
}

\author{
Catriona Macaulay \\ School of Computing \\ University of Dundee \\ catriona@mac.com
}

\author{
Nick Hine \\ School of Computing \\ University of Dundee \\ nhine@computing.dundee.ac.uk
}

\begin{abstract}
Persuasion has played an important role in human life since it began. In the early 1990s a new approach called persuasive design was introduced into the $\mathrm{HCl}$ field. Persuasive design recognised that persuasion knowledge could be applied to the design and development of a variety interactive applications for changing people's attitudes and behaviours. However, the field remains very young, with the transfer of persuasion knowledge to interaction designers particularly limited. This paper presents work towards the design of a toolkit to make this knowledge more accessible to designers during the design lifecycle. The paper introduces our work towards a "Persuasion Knowledge Toolkit" (PToolkit). We present the key body of work undertaken so far; the generation of requirements for such a toolkit and our exploration of the challenges in making rich bodies of literature around topics such as persuasion accessible to designers.
\end{abstract}

Persuasive Technology, Persuasion Knowledge, Requirements Gathering, qualitative methods, Interview, Workshops, Persona and Scenario.

\section{INTRODUCTION}

In the early 90's BJ Fogg introduced new domain in human computer interaction $(\mathrm{HCl})$ - persuasive technology (PT). PT is defined as the use of interactive applications to change people's attitude and behaviour (Fogg 2003). This approach utilises persuasion knowledge in the design and development of various interactive applications.

Whilst the field initially started with the specific aim for changing people's attitudes and behaviours, the use of persuasive technology has latterly been extended towards more universal and everyday products. For example mobile phones have been designed to remind users of their personalised exercise regime, web sites used for shopping for groceries provide home delivery services thus reducing user's fuel usage and time consumption, and social networking is used to offer support to people during difficult times. In fact, nowadays those situations are so common that persuasive technologies have become part of most people's everyday experiences. Therefore, making the rich body of knowledge about persuasion accessible and appropriate for people involve in designing persuasive applications is growing in importance.

\section{BACKGROUND}

\subsection{Persuasive Technology}

During the early years of the computing era, the idea that a computer system could be designed to impact people's beliefs or behaviours would have been unfamiliar, and possibly unwelcome. Computer applications existed for far more prosaic purposes - they were designed to their users achieve 'tasks', not help someone else altogether change the user, However, as technology evolved and increasingly dominated people's activities, the idea that applications could be used for persuasion gradually become an important and even acceptable prospect. Without a doubt embedding persuasion into the design of interactive application is a new challenge for designer. Many questions may arise:

- What persuasion knowledge is suitable, possible and appropriate for designers to use?

- Is the established canon of knowledge about persuasion stable, realistic and significant enough to be used with the new media/technology?

- How can we make the knowledge accessible to non-specialists?

- Even if we can make it accessible, will it be usable by designers? 
Until now, few attempts have been made to guide designers in designing with persuasion in mind, albeit with some notable exceptions (Fogg 2009)(Lockton et al. 2009) . Whilst these are a good start, the field falls significantly short of for example efforts to transfer highly specialised knowledge about ergonomics or accessibility to the designers of computers applications.

\subsection{Persuasion Knowledge Toolkit}

The Persuasion Knowledge Toolkit (PToolkit) is an attempt to transfer persuasion knowledge to designers. Ideally the PToolkit should be used by designers during the design ideation phase of the design lifecycle. It aims to guide and inspire designers during design ideation process by providing them with the knowledge required to make appropriate and better grounded design choices for the particular persuasion challenge they are addressing.

Our requirements generation research for the PToolkit was segmented into 3 stages:

(i) During the first stage, we were dealing with the challenge of what knowledge is needed by the designer (since we recognise that not all knowledge is necessarily appropriate). Investigations at this stage revealed that there is a significant gap in knowledge accessibility that needs to be addressed. The knowledge is mainly trapped in books and journal articles and transfer to the design community is still far from satisfactory (Torning et al. 2009)(Bolchini et al. 2008). We drew persuasion knowledge from various academic resources that could be very useful in persuasive design.

(ii) In the second stage, we are working on how the knowledge should be given to the designer in a way that is suitable for them and fit in their working practice. In this stage we will design and develop the PToolkit prototype. Ideally this prototype will be used to transfer persuasive knowledge to the designer in order to inspire the persuasive design idea.

(iii) Evaluation of PToolkit regarding issues on knowledge transfer appropriateness. It may indrawn insight in bridging the gap of knowledge transfer between research community and design community.

At the moment, we are on the second stage and working on design and development of PToolkit. As PToolkit meant for designer, it's design and development apparently needs us to understand designer, design activity or process and how the complex knowledge of persuasion (non-design knowledge) is used within the design activity.

\section{REQUIREMENT GATHERING}

Designing PToolkit will be a very big challenge for us. Firstly it is designed by a non-designer (researcher) for designers who are widely known as people with endless imagination and creativity. Secondly, it requires deep understanding of how designers design in order to ensure that the PToolkit fits well in design the environment

Understanding designer and design activity are very crucial in this phase. Designers are mostly living in active mode (practical) and the design process usually deals with ill-structured design problems. This has led us to investigate a completely different working setting. As a result we decided to employ two qualitative data gathering methods to address the situations.

To gather the data, we're using (i) interview method to gain insight into designer's personality and working nature (ii) conduct a series of design workshops to help us understand the design process and how the persuasion knowledge (nondesign knowledge) is used and impact the overall designer's experienced.

\subsection{Interviewing designers}

Interview is an established method that has been widely used during data gathering. It provides insight about designer and their work. The main objective of this interview is to understand who is designer and how they design.

At the moments we have already conducted three interview sessions with designers. The interviews were done separately and last for approximately 45 minutes for each session. We used semi structured interview format and open ended questions. Two interview sessions were held in the designer studio, which provided us opportunity to observe their working environment. This has helped us to obtain some culturally rich data. However, for some technical reason another interview was done in researcher's meeting room.

During the interview we were recording the voice data and took some notes. This recorded data was then securely kept, transcribed and discussed in later stages.

\subsection{Design Workshops}

The design workshops used the participatory design approach with some controversial issues as part of the design challenge (tasks). This design challenge is important in order to create an engaging, fun and lively design environment. It is essential to ensure that the design workshop is fun and able to generate innovative ideas. 
The objectives of these workshops are;

(i) Understanding design process / flows.

(ii) Understanding the impact of persuasion knowledge (non-design knowledge) in the design process.

\subsubsection{Design Workshop Framework}

The design workshop was segmented into three sessions. The first two sessions were conducted in one day that required the participants' time commitment for approximately 4 hours.

\section{These sessions were divided into 3 tasks:}

(i) The first task required participants to brainstorm and then come out with a conceptual design of a campaign web page that was able to persuade parents to believe that video games are good for their children. This task took approximately 45 minutes. Participants were provided with materials such as paper, pencils, pens, colours, and post it notes to help them to illustrate and organise the design ideas.

(ii) The second task required participants to illustrate their design processes carried out in the first design task.

(iii) The third task was a reflection session in which all participants actively discussed about the previous design tasks and other design related issues.

The third design workshop session will be conducted within a few weeks time as they will be assigned to design with the assistance of the PToolkit prototype. As at the moment this prototype is still under development, the design workshop will take place sometimes later.

\subsubsection{Implementation}

\section{(i) Group 1}

Group 1 consists of two designers and they decided to produce an online game for children (figure1). This game requires the children to solve some puzzles that are arranged in various forms in order to invade a Castle. The puzzles expect children to take part in various activities in mathematics, reading, language, resources management, ethics and morals. Children are then given rewards when they have solved the puzzle. The participants believed that this puzzle was able to increase the children's skill. The results of their achievement and skill throughout the gameplay can be gathered, printed and shared online with the parents. Parents may be influenced by the results of their kids, and believe that video games are good for children.

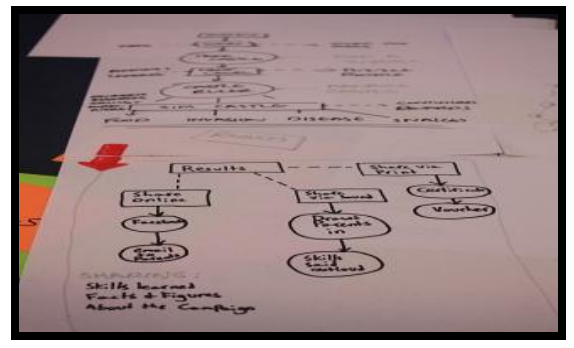

Figure 1: Group 1's Conceptual Design

\section{(ii) Group 2}

Group two consists of three designers that designed a website for persuading parents about the benefits of playing video games. Initially, this group had some difficulties in deciding how the conceptual design should be. They had some arguments about the role and perspectives of the persuasion campaign assigned to them. They were discussed a few roles from different perspectives such as Non Government Organisation (NGO), government or the marketing department of a video game company. They decided to take the role of a video game company, while at the same time promoting the benefits of playing video games to kids. The participants has designed a web page that specifically mentioned the elements of persuasion such as forum and discussion for social networking, video game rating given by parents, and incentives for buying game. This group also considered applying some creative and good user interface design to encourage effective use by mentioning about "friendly colour palette and easy to use user interface".

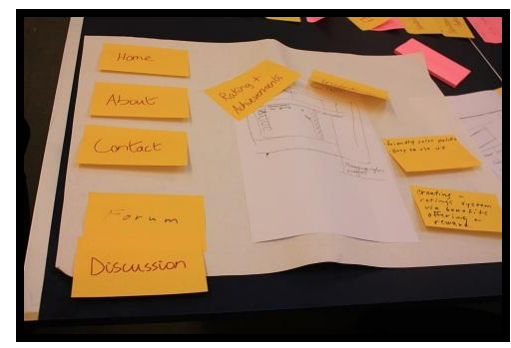

Figure 2: Group 2 Conceptual Design

\section{(iii) Individual Design Process}

The second part of the design workshop session required the participants to illustrate their design process (figure 3 ). Design activity has been widely discussed both in practical and academic setting. Interestingly the discussion is still going on as design is an evolving area and requires designer to adapt the design activity based on various factors 
including people, group dynamic, technology and many more.

Two participants admitted that their design process resides in their head. The task of Illustrating the process onto the paper is quite difficult, however it worth especially into reflecting on their own understanding. Another participant said that he never has his own design process, however learnt a lot about it in the formal design class and other designers. However, he acknowledged that having the design process is important as it is able to guide throughout design activity. Well documented design process makes design activities and documentations (if needed) smoother especially when the design is done for large organisation.

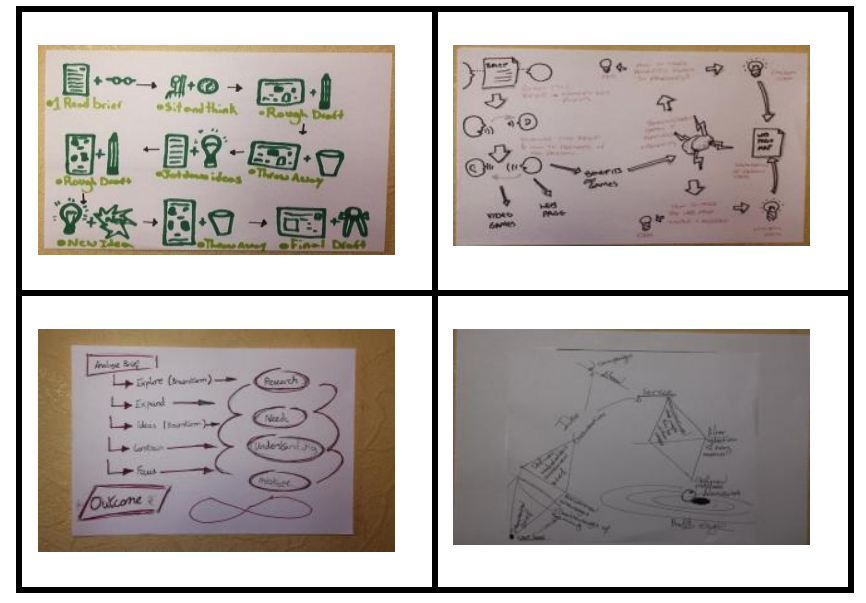

Figure 3: Some individual design process

\subsection{Challenges}

\subsubsection{Participants}

Collaborating human participants could bring a huge challenge for any research. We also faced difficulties in involving the designers that working in a company or doing freelance. Most of them were reluctant to participate in this research for various reasons such as time consuming, not interested in the persuasion subjects and the research has no obvious benefit to them. Therefore, we were decided to invite student that has experienced in design fields. The participants involved in this research are master students that currently studying in design of ethnography in University of Dundee. Most of them have more than 4 years design experienced and working in various design setting before continue their studies here.

\subsubsection{Incentives for commitment}

At the beginning only few designers interested to participate in the design workshop as it requires quite a long time commitment. Therefore, we offered few incentives in order to attract participants to take part such as, (i) break the session into shorter period of time (2 hours in one day), (ii) allow them to have the written report based on the results collected, and (iii) provide them with gift card as an appreciation of time being spent in the design workshop.

\subsubsection{Design Tasks}

It is also important that the design tasks created have to fulfil the research objectives, challenging and fun. It is to ensure that the workshops will provide researchers with the answers of what they are looking for and at the same time participants are able to engage and honestly create some innovative design. As this workshop is only a mock up design activity, the challenge should be planned closely towards the real design practices.

\section{DISCUSSION AND INTERPRETATION}

\subsection{Design Issues}

\subsubsection{Design Process}

Understanding the design process is important to lead us to the context of when and how the persuasion knowledge is applied during design process. Various design process illustrations that were collected from each designer proved that (i) design process is individuality and (ii) formal design methods suggested by various literature or text books are rarely followed. Their design processes were analysed and generalised into figure 4 .

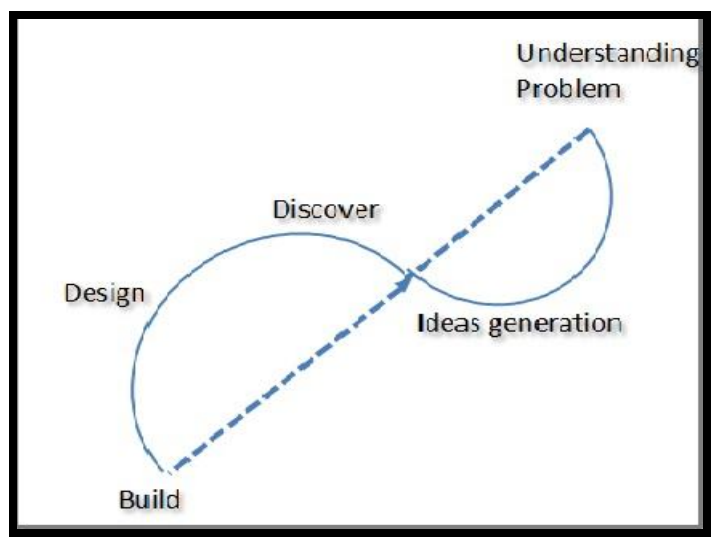

Figure 4: Design process

The design process has a few stages of:

- Understanding problem

- Ideas generation/design ideation

- Discover

- Design

- Build 


\subsubsection{Persuasion Knowledge}

This section will describe on two aspects of persuasion knowledge were observed throughout the workshop. First aspect was on how participants used the persuasion knowledge in the design process. It was observed that:

(i) Persuasion knowledge is used mainly during the design ideation and discovers stages. They brainstormed and discussed on how to best influence the parents using various persuasion element.

(ii) Participants were quite familiar with some of the persuasion knowledge as they mentioned about it (rewards, rating, and social networking) during the design process.

(iii) Both groups were used the well-known persuasion elements. These may be because of unfamiliarity of other types of persuasion elements that also good in persuading people.

Second aspect observed was on the attitudes of participants toward the persuasion concept. Generally persuasion is associates with the negative implication as it literally meant to persuade someone to do or believe something. On the contrary a participant mentioned that persuasion may be very helpful in situation whereby controlling people behaviour is most needed. This controlling behaviour is necessary to ensure that intended process goes smoothly. Another participant said that the design itself is about persuading people. $\mathrm{He}$ added that any design should able to attract the intended audience. Embedding persuasion in design of interactive artefacts seems an awkward concept and could be misleading. However most of them agreed about the benefits of persuasion and its importance in design of interactive application.

\subsubsection{Idea Generation}

All the participants agreed that most ideas will be generated during brainstorming sessions. They believed that discussion allows the idea to be developed more profoundly. To generate the ideas designer needs to understand the task given which is usually done during the briefing session. Then they need to figure out the design objectives which afterwards will lead them to think of several alternative designs. At this point, designers need to make some interpretations on the tasks. The greater space of interpretation are allowed, the outcome generated could be different from one to another. This interpretation also allows designers to think of different alternative solutions.

The ideas are usually come in several ways:

(i) Using external resources for finding information such as internet, books and magazine could generate ideas. (ii) Looking at others people that are working in similar context. Other people's ways of solving problems are able to inspire the design idea.

(iii) Some of them also admitted that the ideas do come naturally. "We're working with imagination, and our head is full with ideas. It is our speciality and we used it to earn for living" said one of them.

\subsection{Modelling user: Persona and Design Scenario}

The interview and design workshops were also used to design and develop the persona and design scenario. The persona was given a name, characteristics, occupation, hobby, and gender. It was made up so to make it as realistic as real person should be. This persona acts as 'target user' (Kuroda et al. 2004) who will use the PToolkit later

This virtual person then was given a story regarding their working context. This context was significant towards the understanding of how target user works with the tool to achieve their goal (Ljungblad et al. 2006) . We created a story for this persona about his design attitude and activity. This story (design scenario) was generated by analysing answers from the interviewed question such as "how you design", "how the idea comes during the design process", and "how do you get inspired". The design workshop then refined the scenario by observing how designer accomplished the design task given.

There are three types' of persona designers that has been created and each of this represent types of designers that we have seen, talked and worked with during the interview and design workshop sessions.

(i) Jack: Jack is a freelance designer that has designed lots of both online and offline artefacts. His goal in design is to understand the real problem faced by his client. He starts the design process by doing some research and reading the related problem area. In finding any information he easily gets attracted with things that catchy and design with great care. $\mathrm{He}$ also likes to observe the environment to look on how people's deal with their problem. Those will inspire him in generating great idea in design.

(ii) Ryan: Ryan is web and graphic designer which is currently working in a design company. His design goal is to understand what his client really needs and solve the design problems effectively and efficiently. $\mathrm{He}$ gets inspired by looking at design examples from famous designers, website, 
pictures/graphics online or offline. He thinks that understanding how other designers solved some design problem and adapted whenever necessary is the effective way to find quick solutions.

(iii) Matt: Matt has designed various artefacts and events since 4 years ago. His design goal is to understand and solve the design problem effectively. In design work, Matt prefers to work individually. He does respect other people suggestion, however working alone provides more room for being creative and allows him to apply the design idea freely. His approach in design is mainly by observing environment/situation and looks how it could be implemented in the design problem he is working on. He always found out that effective design solutions can be achieved by looking on practicality rather than looking on theoretical point of view.

\subsection{Design Implication: PToolkit Prototype}

PToolkit prototype will take a form of an interactive application. It serves as a platform to help us gain insight of persuasion knowledge transfer from academic community to design community.

We are using persona and scenario to design and developed the PToolkit prototype. There are two great aspects of persona and scenario during design and development that has influenced us so much; (i) provides concept and context of what and how the prototype (application) should be in the beginning (ii) acts as evaluation tools to verify on the application's functionality at the end of design process.

There are few most important areas that particularly suggested by persona and scenario that would affect the designs of PToolkit:

(i) The knowledge of persuasion need to be made obvious in order to allow deeper understanding.

(ii) The persuasion knowledge's information architecture which currently is complex has to be structured accordingly to make it more accessible.

(iii) The use of appropriate persuasive technology examples could be use to inspire designers and increase their understanding.

(iv) Navigation need to be carefully design in order to provide good experience and appropriate knowledge for designers.

(v) Interface design should be user friendly and attractive in order to encourage designer's engagement in exploring the possible and appropriate use of persuasion knowledge.

\section{CONLUSION AND FUTURE WORKS}

The chosen qualitative methods in requirement gathering phase have provided rich and insight particularly on designers, and design process. It also allowed us to understand the value of persuasion knowledge and how it can be used effectively during design process.

We are currently designing the PToollkit prototype to be used in the third design workshop. This workshop will make full use of participatory design approach which will help in refining the PToolkit prototype. We are supposed that the process of PToolkit prototyping pave the deep understanding on persuasion knowledge transfer to designer community.

\section{REFERENCE}

Bolchini, D., Garzotto, F. \& Paolini, P., 2008. Valuedriven design for "infosuasive" web applications. Proceeding of the 17th international conference on World Wide Web - WWW '08, 4(1), p.745. Available at:

http://portal.acm.org/citation.cfm?doid=1367497.13 67598.

Fogg, B.J., 2003. Persuasive Technology: Using Computer to Change What We Think and Do, San Francisco USA: Morgan Kaufman.

Fogg, B.J., 2009. The Behavior Grid: 35 Ways Behavior Can Change. In Persuasive'09. pp. 1-5.

Kuroda, K. et al., 2004. Smart-media design process with scenario based modeling. 2004 International Symposium on Applications and the Internet Workshops. 2004 Workshops., pp.467472. Available at:

http://ieeexplore.ieee.org/lpdocs/epic03/wrapper.ht $\mathrm{m}$ ?arnumber $=1268675$.

Ljungblad, S. et al., 2006. Designing Personal Agent with Persona. Development, pp.575-580.

Lockton, D. et al., 2009. Influencing Interaction: Development of the Design with Intent Method. In Persuasive 09. pp. 1 - 8.

Torning, K., Hall, C. \& Oinas-kukkonen, H., 2009. Persuasive System Design : State of the Art and Future Directions. Information Systems. 